\title{
Reciprocal recurrent selection effects on the genetic structure of tropical maize populations assessed at microsatellite loci
}

\author{
Luciana Rossini Pinto ${ }^{1}$, Maria Lucia Carneiro Vieira ${ }^{1}$, Claudio Lopes de Souza Jr. ${ }^{1}$ \\ and Anete Pereira de Souza ${ }^{2}$ \\ ${ }^{1}$ Universidade de São Paulo, Escola Superior de Agricultura "Luiz de Queiroz", \\ Departamento de Genética, Piracicaba, SP, Brazil. \\ ${ }^{2}$ Universidade Estadual de Campinas, Centro de Biologia Molecular e Engenharia Genética, \\ Departamento de Genética e Evolução, Campinas, SP, Brazil.
}

\begin{abstract}
A modified reciprocal recurrent selection (RRS) method, which employed one cycle of high-intensity selection, was applied to two tropical maize (Zea mays L.) populations, BR-105 and BR-106, originating the improved synthetics IG-3 and IG-4, respectively. In the present study the effects of this kind of selection on the genetic structure of these populations and their synthetics were investigated at 30 microsatellite (SSR) loci. A total of 125 alleles were revealed. A reduction in the number of alleles was observed after selection, as well as changes in allele frequencies. In nearly $13 \%$ (BR-105) and 7\% (BR-106) of the loci evaluated, the changes in allele frequencies were not explained, exclusively due to the effects of genetic drift. The effective population sizes estimated for the synthetics using 30 SSR loci were similar to those theoretically expected after selection. The genetic differentiation $\left(G_{S T}\right)$ between the synthetics increased to $77 \%$ compared with the original populations. The estimated $R_{S T}$ values, a genetic differentiation measure proper for microsatellite data, were similar to those obtained for $\mathrm{G}_{\mathrm{ST}}$. Despite the high level of selection applied, the total gene diversity found in the synthetics allows them to be used in a new RRS cycle.
\end{abstract}

Key words: population genetics, plant breeding, molecular markers.

Received: October 15, 2002; Accepted: June 26, 2003.

\section{Introduction}

Important increases in maize productivity have been obtained since the beginning of the last century because of the development of inbreeding and hybridization methods outlined by Shull (Crow, 1998). Currently, most maize breeding programs are based on hybrid production. The development of inbred lines and hybrids is very much related to the frequency of favorable alleles, which can be increased via recurrent selection (Hallauer and Miranda 1988). In this kind of selection, populations and inbred lines are developed to be crossed and to form superior hybrids. In the reciprocal recurrent selection method (RRS), genotypes from two populations are evaluated in reciprocal crosses, where each population is used as the other's tester. The improved populations are generated by intermating superior genotypes of each population that present the best combining abilities with the reciprocal population (Souza Jr., 1998).

Similarly to selection methods, RRS causes changes in the allele frequencies, levels and distribution of the ge-

Send correspondence to Maria Lucia Carneiro Vieira. E-mail: mlcvieir@esalq.usp.br. netic variability, and, consequently, in the genetic structure of the populations. The use of inadequate population sizes leads to the loss of genetic variability due to genetic drift effects. Such loss can limit long-term RRS programs (Guzman and Lamkey, 1999, 2000). For this reason, highintensity selection has been avoided in conventional RRS. However, Rezende and Souza Jr. (2000) applied one cycle of high-intensity RRS in the tropical maize populations BR-105 and BR-106 and, despite the negative drift effects on the improvement of the populations per se, the interpopulation genetic variances were not significantly affected.

Molecular markers are promising for the investigation of all these changes. Labate et al. (1999) described significant changes in the allele frequencies at most maize loci after 12 cycles of RRS using the RFLP (restriction fragment length polymorphism) procedure, and genetic drift hypothesis was rejected by the Waples' neutrality test (Waples, 1989a). Koeyer et al. (2001) identified genomic regions containing favorable alleles using 97 RFLP loci to monitor genetic changes in a long-term recurrent selection program in oats. 
Currently, microsatellite markers are commonly employed for the analysis of plant population genetic structure because of their co-dominant nature and high informativeness. These markers represent non-coding DNA regions composed of small motifs of 1 to 6 nucleotides repeated in tandem, which are under mutation rates higher than those observed at the rest of genome (Jarne and Lagoda, 1996). Because of this, Slatkin (1995) proposed a genetic differentiation measure $\left(\mathrm{R}_{\mathrm{ST}}\right)$ similar to Wright's (1951) $\mathrm{F}_{\mathrm{ST}}$ and Nei's (1973) $G_{\mathrm{ST}}$ that seems more adequate for microsatellite data analysis.

This study evaluated at the molecular level the effects of a high-intensity RRS cycle on the genetic structure of two important tropical maize populations, BR-105 and BR-106. Microsatellite loci were used to estimate (i) the change in allele frequencies after one cycle of selection, (ii) the genetic differentiation between the populations BR-105 and BR-106, and between their synthetics IG-3 and IG-4, expressed as $\mathrm{G}_{\mathrm{ST}}$ and $\mathrm{R}_{\mathrm{ST}}$, and (iii) the effective population sizes of the synthetics.

\section{Material and Methods}

\section{Development of plant materials}

Two maize populations, a Thai (BR-105) and a Brazilian composite (BR-106) and their synthetics were analysed. BR-105 and BR-106 were previously submitted to one cycle of high-intensity RRS (2.0 and $2.5 \%$, respectively) using $400 \mathrm{~S}_{3}$ lines. These lines were crossed with the opposite population and superior interpopulation half-sib (HS) progenies were identified. Eight $\mathrm{S}_{3}$ lines $(2.0 \% \times 400)$ derived from BR-105 and ten $\mathrm{S}_{3}$ lines $(2.5 \% \times 400)$ derived from BR-106, both related to the selected interpopulation HS progenies, were intercrossed in a diallel mating design within each population to develop IG-3 and IG-4 synthetics, respectively (Figure 1). The synthetics IG-3 and IG-4 resulted from intercrossing of $\mathrm{S}_{3}$ lines i.e. they are the product of random mating of the alleles of eight lines derived from BR-105, and ten lines derived from BR-106, respectively. Considering that the effective population size $(\mathrm{Ne})$ of each $\mathrm{S}_{3}$ is approximately 0.57 , the $\mathrm{Ne}$ of IG-3 and IG-4 are 4.56 and 5.71 with inbreeding coefficients of $10.9 \%$ and $8.75 \%$, respectively (Rezende and Souza Jr., 2000).

\section{DNA extraction and SSR assays}

One hundred randomly chosen seeds were taken from each population and synthetics. Leaf tissues collected from 35 day-old plants were lyophilized, ground by a mechanical mill, and stored at $-20{ }^{\circ} \mathrm{C}$. Total genomic DNA was extracted from $300 \mathrm{mg}$ of lyophilized tissues using a CTAB procedure (Hoisington et al., 1994). PCR reactions were performed in a $20 \mu \mathrm{L}$ final volume containing $40 \mathrm{ng}$ of template DNA, $0.2 \mu \mathrm{M}$ of each forward and reverse primer, $100 \mu \mathrm{M}$ of each dNTP, $2.0 \mathrm{mM} \mathrm{MgCl} 2,0.5$ unit Taq DNA polymerase (Gibco-BRL), $10 \mathrm{mM}$ Tris- $\mathrm{HCl}$ and $50 \mathrm{mM}$

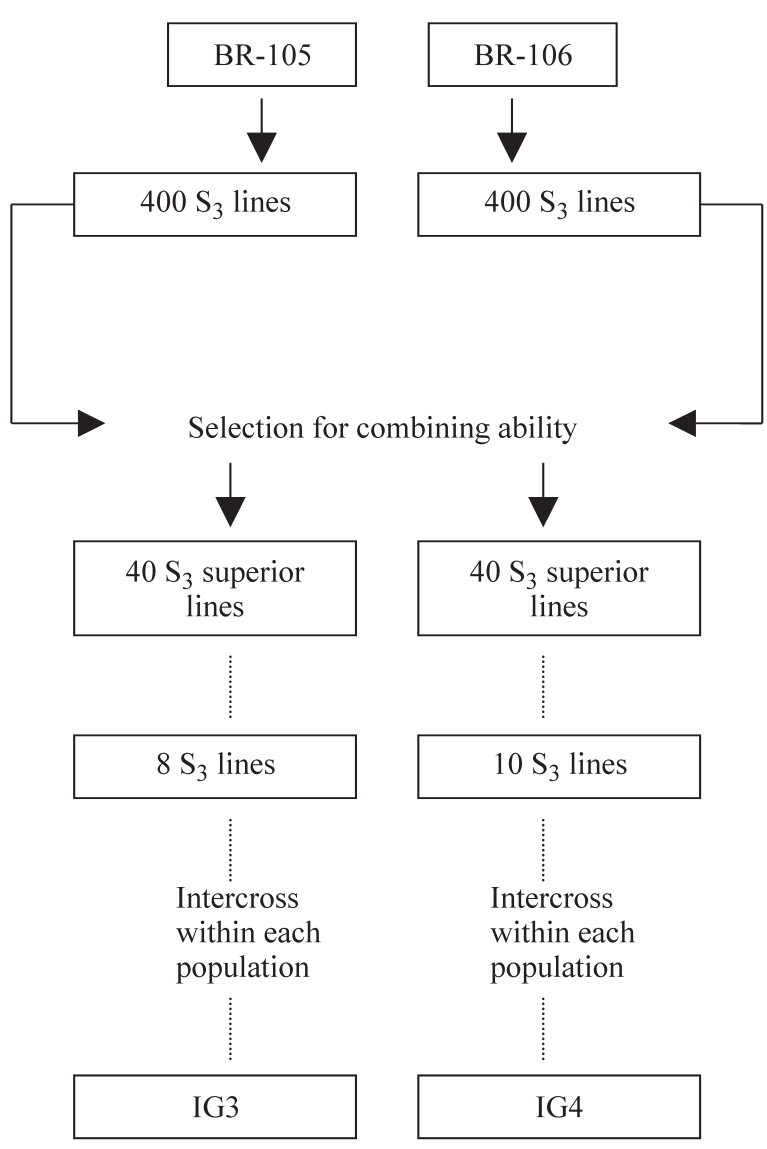

Figure 1 - Description of the modified RRS in which a high-intensity of selection was applied to BR-105 and BR-106 giving rise to the synthetics IG3 and IG4.

$\mathrm{KCl}$. Reactions were run in a PTC-100 thermocycler (MJ Research) using the PCR cycling conditions described by Ogliari et al. (2000). Thirty primer pairs located at least in one maize chromosome were used to survey the genetic polymorphism. Amplification products were separated by electrophoresis on $3 \%$ agarose gels (50\% agarose metaphor FMC-Bio products: $50 \%$ agarose Gibco-BRL) in TBE buffer (0,09 M Tris, 0,09 M boric acid, 2 mM EDTA). Gels were photographed under UV light after ethidium bromide staining. The sizes of the fragments were calculated by comparison with 50 and 100 bp ladders.

\section{Statistical analysis}

\section{Allele frequencies}

Individuals were genotyped in terms of their alleles and respective SSR loci, defined by a primer pair (forward/reverse). Allele frequencies were calculated using the BIOSYS-1 program (Swoffford and Selander, 1991). To test the hypothesis of identical distribution of the allele frequencies, an exact test for population differentiation was performed with the TFPGA program (Miller, 1997). The neutrality test (Waples, 1989a; Labate et al., 1999) was applied to each locus to verify whether the changes in allele 
frequencies after one cycle of RRS could be exclusively attributed to the genetic drift effects.

\section{Diversity distribution}

The distribution of gene diversity was conducted according to the model proposed by Nei (1973), in which the total genetic diversity mean $\left(\mathrm{H}_{\mathrm{T}}\right)$ is partitioned in two components: the gene diversity mean within population $\left(\mathrm{H}_{\mathrm{S}}\right)$, and between populations $\left(\mathrm{D}_{\mathrm{ST}}\right)$. The proportion of total gene diversity $\left(\mathrm{G}_{\mathrm{ST}}\right)$ between population, or genetic differentiation, was calculated as $\mathrm{G}_{\mathrm{ST}}=\mathrm{D}_{\mathrm{ST}} / \mathrm{H}_{\mathrm{T}}$. To better understand the behavior of gene diversity after one cycle of selection, total gene diversity was performed separately for populations (before selection - $\mathrm{C}_{0}$ ), and for synthetics (after selection $-\mathrm{C}_{1}$ ), as well as for the combinations BR-105 vs. IG-3, and BR-106 vs. IG-4 using the FSTAT program (Goudet, 1995). Genetic differentiation was also estimated by using the $\mathrm{R}_{\mathrm{ST}}$ statistics, as the fraction of total variance in the allele size (in base pairs) that occurs between populations, using the RSTCALC package (Goodman, 1997).

\section{Effective population size}

IG-3 and IG-4 effective population sizes ( $\mathrm{Ne}$ ) were estimated according to the Waples method (1989b), based on Plan II, where the individuals are taken before the reproduction event, and not replaced. Considering $N_{0}$ and $N_{t}$ as the respective sampling sizes at the two sampling events, $\mathrm{t}$ the time between the two sampling events, and $\hat{\bar{F}}_{C}$ the weighted standardized variance in allele frequencies, $\mathrm{Ne}$ is given by

$$
\hat{N} e=\frac{t}{2\left(\hat{\bar{F}} c-\frac{1}{2 N_{0}}-\frac{1}{2 N_{t}}\right)}
$$

The standardized variance in allele frequencies for each locus $(\hat{F} c)$ was calculated using the expression proposed by Nei and Tajima (1981):

$$
\hat{F}_{C}=\frac{\sum_{u=1}^{k}\left(p_{u}-p_{u}^{\prime}\right)^{2}}{\sum_{u=1}^{k} \frac{\left(p_{u}+p_{u}^{\prime}\right)}{\left(2-p_{u} p_{u}^{\prime}\right)}}
$$

where $p_{u}$ and $p_{u}^{\prime}$ are the frequencies of the $u$ allele at the two sampling events and $k$ is the number of alleles at a locus. For multiple loci, $\hat{\bar{F}}_{C}$ is given by the weighted means of single locus values $(\hat{F} c)$ by the number of alleles at each locus. The $95 \%$ confidence intervals were calculated using the formula:

$$
\mathrm{IC}_{95 \%}, \hat{N e}=\left[\frac{k \hat{N} e}{\chi^{2}, 0.005, k}, \frac{k \hat{N} e}{\chi^{2}, 0.995, k}\right]
$$

based on the number of $k$ independent alleles $\left(\sum\left(k_{j}-1\right)\right)$.

\section{Results}

\section{Allele frequencies}

The 30 loci revealed a total of 125 alleles, 111 occurring in BR-105 and 116 in BR-106 (Table 1). Most of the alleles that were in low frequency in the original populations were lost after one cycle of RRS. Allele reductions were observed in IG-3 (23\%) and IG-4 (17\%). An increase in the number of alleles belonging to the extreme classes of frequencies was detected in both populations after selection (Figure 2). This is a feature of a dispersive process in which the allele frequencies tend towards the limits of zero (lost) or 1 (fixation). The differentiation tests for the allele fre-

Table 1 - Allele frequency distribution $\left(\hat{p}_{u}\right)$ in two maize populations (BR-105 and BR-106) and their synthetics (IG-3 and IG-4) according to the microsatellite locus.

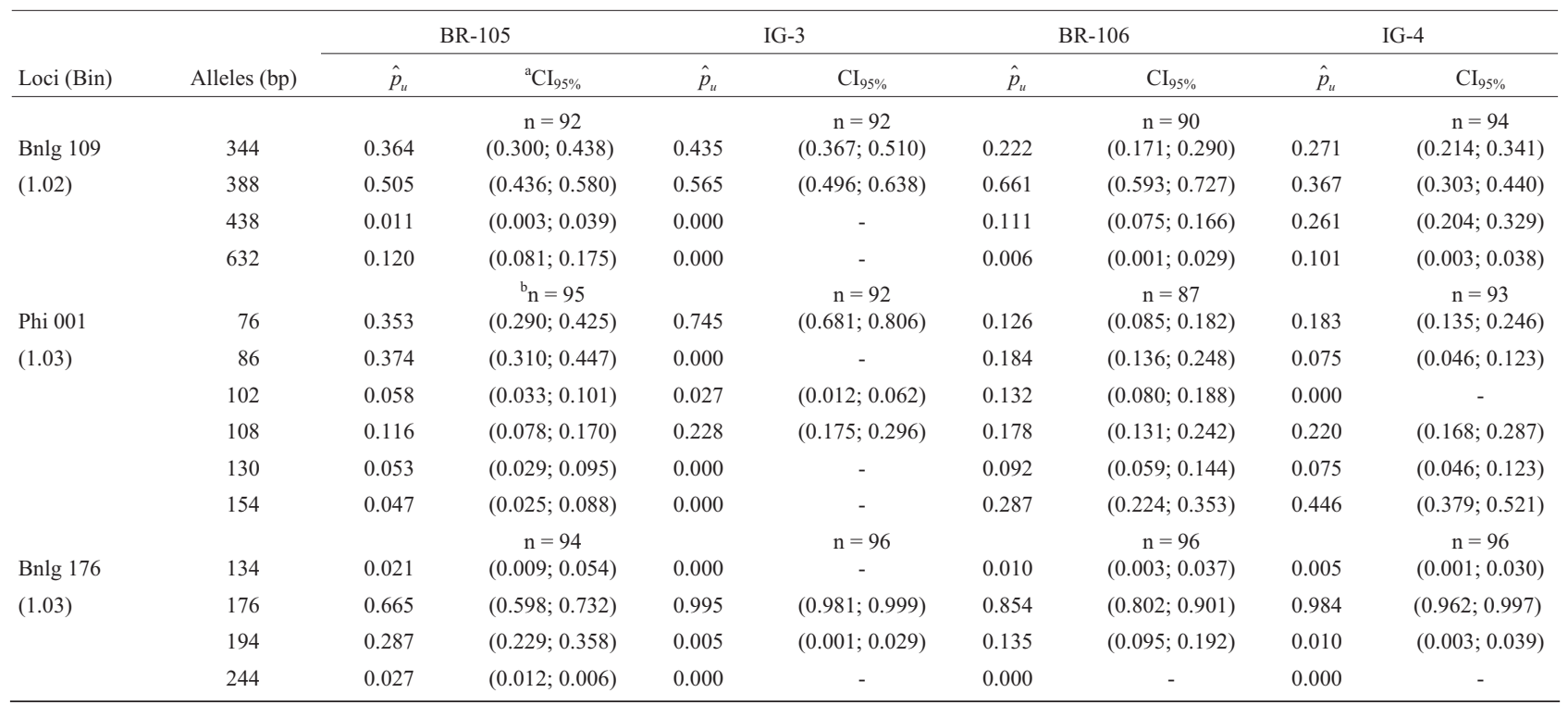


Table 1 (cont)

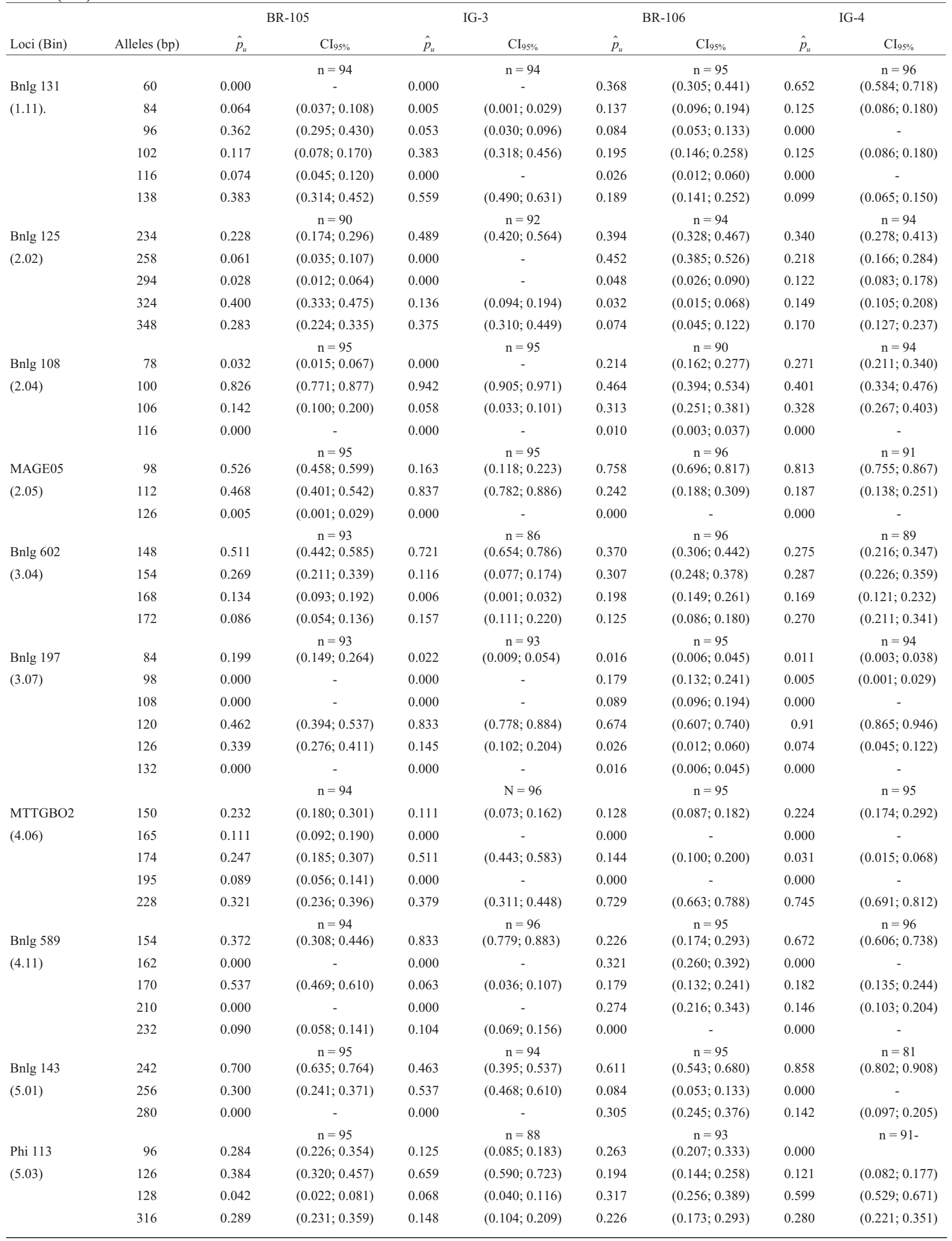


Table 1 (cont)

\begin{tabular}{|c|c|c|c|c|c|c|c|c|c|}
\hline \multirow[b]{2}{*}{ Loci (Bin) } & \multirow[b]{2}{*}{ Alleles (bp) } & \multicolumn{2}{|c|}{ BR-105 } & \multicolumn{2}{|c|}{ IG-3 } & \multicolumn{2}{|c|}{ BR-106 } & \multicolumn{2}{|c|}{ IG-4 } \\
\hline & & $\hat{p}_{u}$ & $\mathrm{CI}_{95 \%}$ & $\hat{p}_{u}$ & $\mathrm{CI}_{95 \%}$ & $\hat{p}_{u}$ & $\mathrm{CI}_{95 \%}$ & $\hat{p}_{u}$ & $\mathrm{CI}_{95 \%}$ \\
\hline & & & $\mathrm{n}=89$ & & $\mathrm{n}=95$ & & $\mathrm{n}=95$ & & $\mathrm{n}=94$ \\
\hline Phi 48 & 188 & 0.033 & $(0.016 ; 0.072)$ & 0.000 & - & 0.000 & - & 0.000 & - \\
\hline \multirow[t]{2}{*}{$(5.07)$} & 196 & 0.815 & $(0.756 ; 0.869)$ & 0.537 & $(0.468 ; 0.609)$ & 0.347 & $(0.285 ; 0.420)$ & 0.431 & $(0.362 ; 0.504)$ \\
\hline & 220 & 0.152 & $(0.107 ; 0.213)$ & 0.463 & $(0.396 ; 0.537)$ & 0.653 & $(0.586 ; 0.720)$ & 0.569 & $(0.501 ; 0.643)$ \\
\hline Bnlg 238 & 134 & 0.353 & $\begin{array}{c}\mathrm{n}=95 \\
(0.290 ; 0.425)\end{array}$ & 0.042 & $\begin{array}{c}\mathrm{n}=95 \\
(0.022 ; 0.081)\end{array}$ & 0.005 & $\begin{array}{c}\mathrm{n}=95 \\
(0.001 ; 0.029)\end{array}$ & 0.000 & $\begin{array}{c}n=94 \\
-\end{array}$ \\
\hline \multirow[t]{5}{*}{$(6.00)$} & 150 & 0.084 & $(0.053 ; 0.133)$ & 0.000 & - & 0.147 & $(0.105 ; 0.206)$ & 0.101 & $(0.066 ; 0.153)$ \\
\hline & 166 & 0.026 & $(0.012 ; 0.060)$ & 0.163 & $(0.118 ; 0.223)$ & 0.063 & $(0.037 ; 0.108)$ & 0.053 & $(0.030 ; 0.096)$ \\
\hline & 184 & 0.105 & $(0.070 ; 0.158)$ & 0.111 & $(0.074 ; 0.164)$ & 0.300 & $(0.240 ; 0.371)$ & 0.309 & $(0.248 ; 0.380)$ \\
\hline & 194 & 0.379 & $(0.315 ; 0.452)$ & 0.653 & $(0.586 ; 0.720)$ & 0.347 & $(0.285 ; 0.420)$ & 0.138 & $(0.097 ; 0.196)$ \\
\hline & 228 & 0.053 & $(0.029 ; 0.095)$ & 0.032 & $(0.015 ; 0.067)$ & 0.137 & $(0.096 ; 0.194)$ & 0.399 & $(0.333 ; 0.473)$ \\
\hline Bnlg 161 & 120 & 0.353 & $\begin{array}{c}\mathrm{n}=95 \\
(0.289 ; 0.425)\end{array}$ & 0.000 & $\begin{array}{c}n=94 \\
-\end{array}$ & 0.000 & $\begin{array}{c}\mathrm{n}=91 \\
-\end{array}$ & 0.000 & $\begin{array}{c}n=93 \\
-\end{array}$ \\
\hline \multirow[t]{6}{*}{$(6.01)$} & 138 & 0.063 & $(0.037 ; 0.108)$ & 0.048 & $(0.026 ; 0.089)$ & 0.000 & - & 0.000 & - \\
\hline & 150 & 0.058 & $(0.033 ; 0.101)$ & 0.149 & $(0.106 ; 0.208)$ & 0.159 & $(0.114 ; 0.221)$ & 0.086 & $(0.054 ; 0.136)$ \\
\hline & 168 & 0.111 & $(0.074 ; 0.164)$ & 0.298 & $(0.238 ; 0.363)$ & 0.214 & $(0.162 ; 0.281)$ & 0.070 & $(0.042 ; 0.120)$ \\
\hline & 176 & 0.379 & $(0.315 ; 0.452)$ & 0.505 & $(0.437 ; 0.579)$ & 0.258 & $(0.201 ; 0.328)$ & 0.360 & $(0.296 ; 0.434)$ \\
\hline & 202 & 0.037 & $(0.018 ; 0.074)$ & 0.000 & - & 0.231 & $(0.177 ; 0.299)$ & 0.091 & $(0.058 ; 0.142)$ \\
\hline & 224 & 0.000 & - & 0.000 & - & 0.137 & $(0.095 ; 0.196)$ & 0.392 & $(0.261 ; 0.395)$ \\
\hline Phi 70 & 80 & 0.548 & $\begin{array}{c}\mathrm{n}=93 \\
(0.479 ; 0.621)\end{array}$ & 0.618 & $\begin{array}{c}\mathrm{n}=93 \\
(0.550 ; 0.688)\end{array}$ & 0.557 & $\begin{array}{c}\mathrm{n}=96 \\
(0.489 ; 0.630)\end{array}$ & 0.526 & $\begin{array}{c}\mathrm{n}=95 \\
(0.458 ; 0.599)\end{array}$ \\
\hline$(6.07)$ & 90 & 0.452 & $(0.384 ; 0.526)$ & 0.382 & $(0.317 ; 0.456)$ & 0.443 & $(0.375 ; 0.516)$ & 0.474 & $(0.406 ; 0.547)$ \\
\hline Bnlg 657 & 84 & 0.000 & $\begin{array}{c}\mathrm{n}=92 \\
-\end{array}$ & 0.000 & $\begin{array}{c}n=94 \\
-\end{array}$ & 0.094 & $\begin{array}{c}\mathrm{n}=96 \\
(0.061 ; 0.144)\end{array}$ & 0.375 & $\begin{array}{c}\mathrm{n}=96 \\
(0.311 ; 0.448)\end{array}$ \\
\hline \multirow[t]{4}{*}{$(7.02)$} & 94 & 0.190 & $(0.141 ; 0.254)$ & 0.298 & $(0.238 ; 0.369)$ & 0.292 & $(0.233 ; 0.361)$ & 0.151 & $(0.108 ; 0.210)$ \\
\hline & 98 & 0.516 & $(0.447 ; 0.590)$ & 0.202 & $(0.152 ; 0.267)$ & 0.302 & $(0.243 ; 0.372)$ & 0.120 & $(0.082 ; 0.174)$ \\
\hline & 104 & 0.114 & $(0.076 ; 0.169)$ & 0.202 & $(0.152 ; 0.267)$ & 0.214 & $(0.162 ; 0.278)$ & 0.182 & $(0.135 ; 0.244)$ \\
\hline & 116 & 0.179 & $(0.131 ; 0.242)$ & 0.298 & $(0.238 ; 0.369)$ & 0.099 & $(0.065 ; 0.150)$ & 0.172 & $(0.126 ; 0.233)$ \\
\hline Bnlg 155 & 92 & 0.763 & $\begin{array}{c}\mathrm{n}=95 \\
(0.698 ; 0.821)\end{array}$ & 0.422 & $\begin{array}{c}\mathrm{n}=96 \\
(0.356 ; 0.495)\end{array}$ & 0.442 & $\begin{array}{c}\mathrm{n}=95 \\
(0.375 ; 0.516)\end{array}$ & 0.511 & $\begin{array}{c}\mathrm{n}=95 \\
(0.442 ; 0.584)\end{array}$ \\
\hline \multirow[t]{5}{*}{ (7.04) } & 108 & 0.026 & $(0.012 ; 0.062)$ & 0.000 & - & 0.289 & $(0.231 ; 0.360)$ & 0.000 & - \\
\hline & 120 & 0.032 & $(0.015 ; 0.007)$ & 0.161 & $(0.171 ; 0.221)$ & 0.084 & $(0.053 ; 0.133)$ & 0.000 & - \\
\hline & 142 & 0.079 & $(0.051 ; 0.131)$ & 0.177 & $(0.130 ; 0.239)$ & 0.032 & $(0.015 ; 0.067)$ & 0.000 & - \\
\hline & 168 & 0.095 & $(0.059 ; 0.144)$ & 0.240 & $(0.186 ; 0.306)$ & 0.142 & $(0.100 ; 0.200)$ & 0.484 & $(0.416 ; 0.558)$ \\
\hline & 188 & 0.005 & $(0.001 ; 0.030)$ & 0.000 & - & 0.011 & $(0.003 ; 0.037)$ & 0.005 & $(0.001 ; 0.029)$ \\
\hline Bnlg 572 & 84 & 0.261 & $\begin{array}{c}\mathrm{n}=90 \\
(0.204 ; 0.332)\end{array}$ & 0.368 & $\begin{array}{c}\mathrm{n}=95 \\
(0.305 ; 0.441)\end{array}$ & 0.541 & $\begin{array}{c}\mathrm{n}=93 \\
(0.469 ; 0.617)\end{array}$ & 0.815 & $\begin{array}{c}\mathrm{n}=96 \\
(0.757 ; 0.868)\end{array}$ \\
\hline \multirow[t]{2}{*}{ (7.07) } & 90 & 0.328 & $(0.265 ; 0.401)$ & 0.105 & $(0.070 ; 0.158)$ & 0.035 & $(0.016 ; 0.074)$ & 0.011 & $(0.003 ; 0.039)$ \\
\hline & 102 & 0.411 & $(0.345 ; 0.487)$ & 0.526 & $(0.458 ; 0.599)$ & 0.424 & $(0.355 ; 0.502)$ & 0.174 & $(0.127 ; 0.237)$ \\
\hline Phi 115 & 90 & 0.505 & $\begin{array}{c}\mathrm{n}=95 \\
(0.437 ; 0.578)\end{array}$ & 0.462 & $\begin{array}{c}\mathrm{n}=93 \\
(0.394 ; 0.537)\end{array}$ & 0.435 & $\begin{array}{c}\mathrm{n}=93 \\
(0.368 ; 0.510)\end{array}$ & 0.578 & $\begin{array}{c}\mathrm{n}=96 \\
(0.510 ; 0.649)\end{array}$ \\
\hline$(8.03)$ & 120 & 0.495 & $(0.427 ; 0.568)$ & 0.538 & $(0.468 ; 0.611)$ & 0.565 & $(0.495 ; 0.637)$ & 0.422 & $(0.356 ; 0.495)$ \\
\hline Bnlg 669 & 108 & 0.368 & $\begin{array}{c}\mathrm{n}=95 \\
(0.305 ; 0.441)\end{array}$ & 0.807 & $\begin{array}{c}\mathrm{n}=96 \\
(0.750 ; 0.861)\end{array}$ & 0.559 & $\begin{array}{c}\mathrm{n}=94 \\
(0.490 ; 0.631)\end{array}$ & 0.826 & $\begin{array}{c}\mathrm{n}=92 \\
(0.769 ; 0.878)\end{array}$ \\
\hline \multirow[t]{7}{*}{ (8.03) } & 114 & 0.047 & $(0.025 ; 0.088)$ & 0.000 & - & 0.043 & $(0.022 ; 0.082)$ & 0.000 & - \\
\hline & 128 & 0.405 & $(0.335 ; 0.473)$ & 0.182 & $(0.135 ; 0.244)$ & 0.011 & $(0.003 ; 0.038)$ & 0.043 & $(0.022 ; 0.084)$ \\
\hline & 144 & 0.095 & $(0.061 ; 0.146)$ & 0.000 & - & 0.005 & $(0.001 ; 0.029)$ & 0.000 & - \\
\hline & 160 & 0.074 & $(0.045 ; 0.121)$ & 0.000 & - & 0.261 & $(0.204 ; 0.329)$ & 0.016 & $(0.006 ; 0.047)$ \\
\hline & 180 & 0.000 & - & 0.000 & - & 0.021 & $(0.009 ; 0.054)$ & 0.000 & - \\
\hline & 200 & 0.011 & $(0.011 ; 0.003)$ & 0.010 & $(0.003 ; 0.037)$ & 0.101 & $(0.066 ; 0.153)$ & 0.114 & $(0.076 ; 0.169)$ \\
\hline & & & $\mathrm{n}=92$ & & $\mathrm{n}=93$ & & $\mathrm{n}=95$ & & $\mathrm{n}=95$ \\
\hline Bnlg 666 & 84 & 0.288 & $(0.229 ; 0.359)$ & 0.258 & $(0.202 ; 0.327)$ & 0.632 & $(0.564 ; 0.700)$ & 0.274 & $(0.214 ; 0.339)$ \\
\hline \multirow[t]{4}{*}{$(8.05)$} & 126 & 0.288 & $(0.229 ; 0.359)$ & 0.199 & $(0.149 ; 0.264)$ & 0.100 & $(0.065 ; 0.152)$ & 0.205 & $(0.153 ; 0.267)$ \\
\hline & 138 & 0.092 & $(0.059 ; 0.144)$ & 0.124 & $(0.084 ; 0.180)$ & 0.111 & $(0.074 ; 0.164)$ & 0.095 & $(0.061 ; 0.144)$ \\
\hline & 166 & 0.293 & $(0.234 ; 0.365)$ & 0.419 & $(0.353 ; 0.494)$ & 0.158 & $(0.114 ; 0.218)$ & 0.426 & $(0.356 ; 0.495)$ \\
\hline & 186 & 0.038 & $(0.019 ; 0.077)$ & 0.000 & - & 0.000 & - & 0.000 & - \\
\hline Bnlg 240 & 112 & 0.100 & $\begin{array}{c}\mathrm{n}=95 \\
(0.065 ; 0.152)\end{array}$ & 0.068 & $\begin{array}{c}n=95 \\
(0.041 ; 0.114)\end{array}$ & 0.313 & $\begin{array}{c}\mathrm{n}=96 \\
(0.252 ; 0.383)\end{array}$ & 0.339 & $\begin{array}{c}\mathrm{n}=96 \\
(0.277 ; 0.410)\end{array}$ \\
\hline (8.06) & 126 & 0.332 & $(0.270 ; 0.403)$ & 0.389 & $(0.325 ; 0.463)$ & 0.042 & $(0.022 ; 0.080)$ & 0.031 & $(0.015 ; 0.067)$ \\
\hline
\end{tabular}




\begin{tabular}{|c|c|c|c|c|c|c|c|c|c|}
\hline \multirow[b]{2}{*}{ Loci (Bin) } & \multirow[b]{2}{*}{ Alleles (bp) } & \multicolumn{2}{|c|}{ BR-105 } & \multicolumn{2}{|c|}{ IG-3 } & \multicolumn{2}{|c|}{ BR-106 } & \multicolumn{2}{|c|}{ IG-4 } \\
\hline & & $\hat{p}_{u}$ & $\mathrm{CI}_{95 \%}$ & $\hat{p}_{u}$ & $\mathrm{CI}_{95 \%}$ & $\hat{p}_{u}$ & $\mathrm{CI}_{95 \%}$ & $\hat{p}_{u}$ & $\mathrm{CI}_{95 \%}$ \\
\hline & 140 & 0.516 & $(0.447 ; 0.589)$ & 0.542 & $(0.474 ; 0.614)$ & 0.375 & $(0.311 ; 0.448)$ & 0.557 & $(0.489 ; 0.629)$ \\
\hline & 158 & 0.053 & $(0.029 ; 0.095)$ & 0.000 & - & 0.271 & $(0.214 ; 0.339)$ & 0.073 & $(0.044 ; 0.119)$ \\
\hline & & & $\mathrm{n}=96$ & & $\mathrm{n}=96$ & & $\mathrm{n}=96$ & & $\mathrm{n}=96$ \\
\hline MCTO2BO8 & 95 & 0.548 & $(0.479 ; 0.620)$ & 0.753 & $(0.688 ; 0.810)$ & 0.635 & $(0.564 ; 0.700)$ & 0.578 & $(0.510 ; 0.649)$ \\
\hline$(9.01)$ & 125 & 0.452 & $(0.385 ; 0.526)$ & 0.247 & $(0.195 ; 0.317)$ & 0.365 & $(0.365 ; 0.304)$ & 0.422 & $(0.356 ; 0.495)$ \\
\hline Phi 65 & 130 & 0.797 & $\begin{array}{c}\mathrm{n}=91 \\
(0.737 ; 0.853)\end{array}$ & 0.346 & $\begin{array}{c}\mathrm{n}=94 \\
(0.285 ; 0.420)\end{array}$ & 0.626 & $\begin{array}{c}\mathrm{n}=91 \\
(0.557 ; 0.697)\end{array}$ & 0.646 & $\begin{array}{c}\mathrm{n}=96 \\
(0.579 ; 0.713)\end{array}$ \\
\hline (9.03) & 150 & 0.203 & $(0.152 ; 0.269)$ & 0.654 & $(0.591 ; 0.725)$ & 0.374 & $(0.308 ; 0.448)$ & 0.354 & $(0.292 ; 0.426)$ \\
\hline Bnlg 127 & 240 & 0.425 & $\begin{array}{c}\mathrm{n}=93 \\
(0.358 ; 0.499)\end{array}$ & 0.707 & $\begin{array}{c}\mathrm{n}=94 \\
(0.642 ; 0.771)\end{array}$ & 0.434 & $\begin{array}{c}\mathrm{n}=91 \\
(0.366 ; 0.509)\end{array}$ & 0.433 & $\begin{array}{c}\mathrm{n}=90 \\
(0.365 ; 0.509)\end{array}$ \\
\hline \multirow[t]{3}{*}{$(9.04)$} & 252 & 0.575 & $(0.506 ; 0.647)$ & 0.293 & $(0.233 ; 0.363)$ & 0.346 & $(0.282 ; 0.420)$ & 0.567 & $(0.496 ; 0.640)$ \\
\hline & 264 & 0.000 & - & 0.000 & - & 0.198 & $(0.147 ; 0.263)$ & 0.000 & - \\
\hline & 276 & 0.000 & - & 0.000 & - & 0.022 & $(0.009 ; 0.055)$ & 0.000 & - \\
\hline Bnlg 292 & 120 & 0.689 & $\begin{array}{c}\mathrm{n}=95 \\
(0.624 ; 0.754)\end{array}$ & 0.984 & $\begin{array}{c}\mathrm{n}=96 \\
(0.963 ; 0.997)\end{array}$ & 0.921 & $\begin{array}{c}\mathrm{n}=95 \\
(0.881 ; 0.956)\end{array}$ & 1.000 & $\begin{array}{c}n=96 \\
-\end{array}$ \\
\hline$(9.06)$ & 152 & 0.311 & $(0.250 ; 0.381)$ & 0.016 & $(0.016 ; 0.045)$ & 0.079 & $(0.048 ; 0.126)$ & 0.000 & - \\
\hline Phi 59 & 144 & 0.000 & $\begin{array}{c}n=96 \\
-\end{array}$ & 0.000 & $\begin{array}{c}n=90 \\
-\end{array}$ & 0.031 & $\begin{array}{c}\mathrm{n}=96 \\
(0.015 ; 0.067)\end{array}$ & 0.000 & $\begin{array}{c}n=90 \\
-\end{array}$ \\
\hline \multirow[t]{2}{*}{$(10.02)$} & 162 & 0.321 & $(0.257 ; 0.380)$ & 0.395 & $(0.328 ; 0.470)$ & 0.318 & $(0.257 ; 0.389)$ & 0.211 & $(0.159 ; 0.278)$ \\
\hline & 171 & 0.679 & $(0.600 ; 0.737)$ & 0.605 & $(0.536 ; 0.677)$ & 0.651 & $(0.584 ; 0.718)$ & 0.789 & $(0.728 ; 0.846)$ \\
\hline Phi 84 & 150 & 0.703 & $\begin{array}{c}\mathrm{n}=91 \\
(0.637 ; 0.769)\end{array}$ & 0.952 & $\begin{array}{c}\mathrm{n}=94 \\
(0.919 ; 0.978)\end{array}$ & 0.705 & $\begin{array}{c}\mathrm{n}=95 \\
(0.640 ; 0.770)\end{array}$ & 0.598 & $\begin{array}{c}\mathrm{n}=92 \\
(0.529 ; 0.669)\end{array}$ \\
\hline \multirow[t]{2}{*}{ (10.04) } & 171 & 0.258 & $(0.201 ; 0.328)$ & 0.005 & $(0.001 ; 0.029)$ & 0.284 & $(0.224 ; 0.353)$ & 0.342 & $(0.279 ; 0.416)$ \\
\hline & 198 & 0.038 & $(0.019 ; 0.078)$ & 0.043 & $(0.022 ; 0.081)$ & 0.011 & $(0.003 ; 0.037)$ & 0.060 & $(0.034 ; 0.104)$ \\
\hline
\end{tabular}

${ }^{\mathrm{a}} \mathrm{CI}_{95 \%}$ : confidence intervals, ${ }^{\mathrm{b}} \mathrm{n}$ : sample sizes, ${ }^{\mathrm{c}} \mathrm{T}$ : total number of alleles.
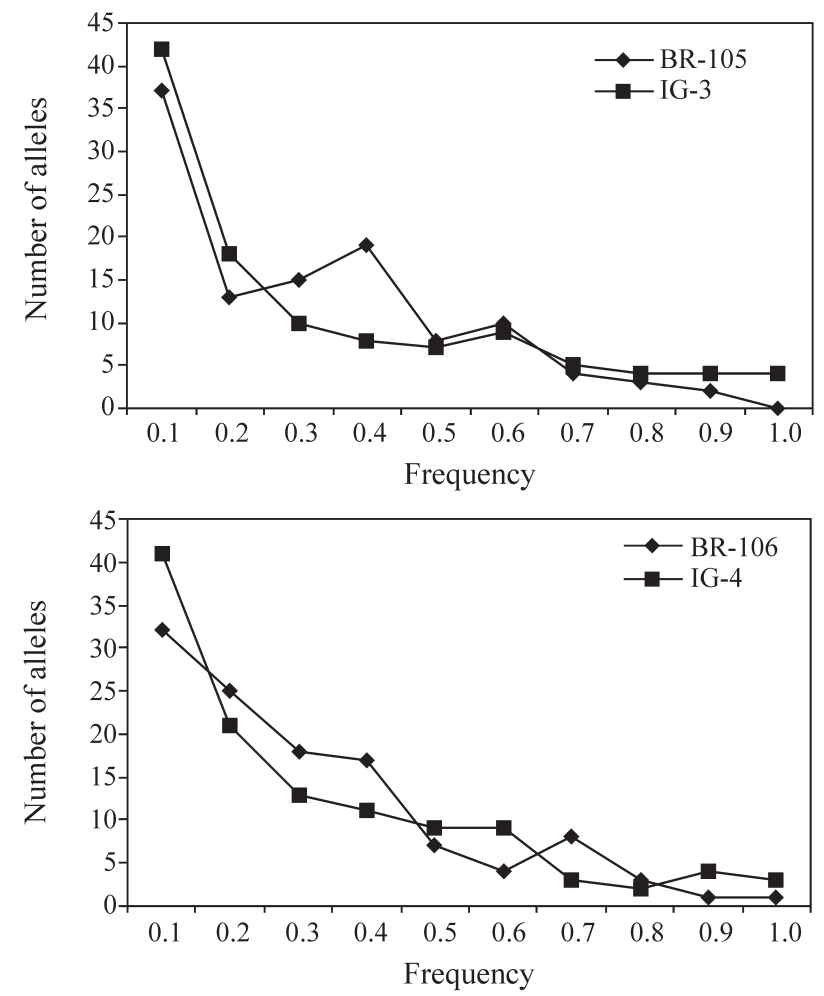

Figure 2 - Allele frequency distribution in the maize populations BR-105 and BR-106 and their synthetics IG-3 and IG-4. quency distribution between the groups (Table 2) were highly significant $(\mathrm{p}<0.01)$. Hence, despite the considerable number of alleles shared between the original populations, these populations differed greatly in allelic frequency. These differences between the original populations and their synthetics could be attributed to the reduced number of lines intercrossed to form the synthetics. The changes in allele frequencies observed after one cycle of RRS $\left(\mathrm{C}_{1}\right)$ were mainly due to the effects of sampling or genetic drift, since the Waple neutrality test was rejected by four loci in BR-105 and two loci in BR-106 (Table 3). These loci represent $13 \%$ and $7 \%$ of the total number in the synthetics IG-3 and IG-4, respectively. Changes in allele frequencies observed for the Phi 65 locus in IG-3 were complementary to IG-4.

\section{Diversity distribution}

The partition of gene diversity before selection $\left(\mathrm{C}_{0}\right)$ showed that most of the gene diversity $(89 \%)$ was within the original populations (Table 4). Similarly, after selection $\left(C_{1}\right), 80.5 \%$ of the total gene diversity found in the synthetics was distributed within them. Contrasting the values of total gene diversity mean $\left(\mathrm{H}_{\mathrm{T}}\right)$ before $\left(\mathrm{C}_{0}\right)$ and after $\left(\mathrm{C}_{1}\right)$ selection, we observed that nearly $10 \%$ was lost, while the mean gene diversity $\left(\mathrm{H}_{\mathrm{S}}\right)$ decreased $18 \%$ between $\mathrm{C}_{0}$ and $\mathrm{C}_{1}$. Comparison of $\mathrm{G}_{\mathrm{ST}}$ values between $\mathrm{C}_{0}\left(\mathrm{G}_{\mathrm{ST}}=11 \%\right)$ and 
Table 2 - Differences in the allele frequency distribution in the four combinations between maize materials.

\begin{tabular}{lcccc}
\hline Combination & BR-105 vs. BR-106 & IG-3 vs. IG-4 & BR-105 vs. IG-3 & BR-106 vs. IG-4 \\
\hline$p$-value & 0.00 & 0.00 & 0.00 & 0.00 \\
df & 60 & 60 & 60 & 60 \\
${ }^{\mathrm{a}} \chi^{2}$ & $539.87^{* *}$ & $515.24^{* *}$ & $561.01^{* *}$ & $489.74^{* *}$ \\
\hline
\end{tabular}

${ }^{\mathrm{a}} \chi^{2}$ test for allele frequency homogeneity: ${ }^{* *}$ significant at $\mathrm{p}<0.01$, df: degree of freedom.

Table 3 - Neutrality test for changes in allele frequencies after one cycle of RRS in the BR-105 and BR-106 maize populations.

\begin{tabular}{|c|c|c|c|c|c|c|c|c|c|}
\hline \multirow[b]{2}{*}{ Locus } & \multirow[b]{2}{*}{ Bin } & \multicolumn{4}{|c|}{ BR-105/IG-3 } & \multicolumn{4}{|c|}{ BR-106/IG-4 } \\
\hline & & $\mathrm{N}_{0}$ & $\mathrm{~N}_{1}$ & $\chi^{2}$ & Df & $\mathrm{N}_{0}$ & $\mathrm{~N}_{1}$ & $\chi^{2}$ & $\mathrm{df}$ \\
\hline Bnlg 109 & 1.02 & 92 & 92 & 0.98 & 3 & 90 & 94 & 5.65 & 3 \\
\hline Phi001 & 1.03 & 95 & 92 & $45.72 * *$ & 5 & 87 & 93 & 4.12 & 5 \\
\hline Bnlg 176 & 1.03 & 94 & 96 & 3.43 & 3 & 96 & 96 & $15.79 * *$ & 4 \\
\hline Bnlg 131 & 1.11 & 94 & 94 & $25.73 * *$ & 4 & 95 & 96 & 0.55 & 5 \\
\hline Bnlg 125 & 2.02 & 90 & 92 & 7.84 & 4 & 94 & 94 & 4.02 & 3 \\
\hline Bnlg 108 & 2.04 & 95 & 95 & 0.73 & 2 & 96 & 96 & 0.10 & 3 \\
\hline MAGE05 & 2.05 & 95 & 95 & 2.35 & 2 & 95 & 91 & 0.17 & 1 \\
\hline Bnlg 602 & 3.04 & 93 & 86 & 2.30 & 3 & 96 & 89 & 0.70 & 3 \\
\hline Bnlg 197 & 3.07 & 93 & 93 & 4.60 & 2 & 95 & 94 & 6.10 & 5 \\
\hline MTTGBO2 & 4.06 & 94 & 95 & 1.55 & 4 & 94 & 96 & 0.48 & 3 \\
\hline Bnlg 589 & 3.07 & 94 & 96 & 1.04 & 2 & 95 & 96 & 2.47 & 3 \\
\hline Bnlg 143 & 5.01 & 95 & 94 & 2.23 & 1 & 95 & 81 & 0.71 & 2 \\
\hline Phi 113 & 5.03 & 95 & 88 & 2.62 & 3 & 93 & 91 & 1.88 & 3 \\
\hline Phi 48 & 5.07 & 89 & 95 & 2.93 & 2 & 95 & 94 & 0.31 & 1 \\
\hline Bnlg 238 & 5.03 & 95 & 95 & 4.71 & 4 & 95 & 94 & 1.47 & 5 \\
\hline Bnlg 161 & 6.01 & 95 & 94 & 1.60 & 5 & 91 & 93 & 1.73 & 4 \\
\hline Phi 70 & 6.07 & 93 & 93 & 0.16 & 1 & 96 & 95 & 0.04 & 1 \\
\hline Bnlg 657 & 7.02 & 92 & 94 & 6.26 & 3 & 96 & 96 & 0.79 & 4 \\
\hline Bnlg 155 & 7.04 & 95 & 96 & 3.91 & 5 & 95 & 95 & 7.82 & 4 \\
\hline Bnlg 572 & 7.07 & 90 & 95 & 1.95 & 2 & 86 & 92 & $27.29 * *$ & 2 \\
\hline Phi 115 & 8.03 & 95 & 93 & 0.06 & 1 & 93 & 96 & 0.85 & 1 \\
\hline Bnlg 669 & 8.03 & 95 & 96 & 2.42 & 5 & 94 & 92 & 8.57 & 6 \\
\hline Bnlg 666 & 8.05 & 92 & 93 & 0.93 & 4 & 95 & 95 & 5.55 & 3 \\
\hline Bnlg 240 & 8.06 & 95 & 95 & 0.11 & 3 & 96 & 96 & 2.09 & 3 \\
\hline МACTO2BO8 & 9.01 & 94 & 95 & 1.40 & 1 & 96 & 96 & 0.14 & 1 \\
\hline Phi 65 & 9.03 & 91 & 94 & $10.48 * *$ & 1 & 91 & 96 & 0.02 & 1 \\
\hline Bnlg 127 & 9.04 & 93 & 94 & 2.74 & 1 & 91 & 90 & 4.62 & 3 \\
\hline Bnlg 292 & 9.06 & 95 & 95 & 3.35 & 1 & 95 & 96 & 0.88 & 1 \\
\hline Phi 59 & 10.02 & 95 & 95 & 0.21 & 1 & 96 & 90 & 0.55 & 2 \\
\hline Phi 84 & 10.04 & 91 & 94 & $26.66 * *$ & 2 & 95 & 92 & 3.25 & 2 \\
\hline
\end{tabular}

** Significant at 0.01 level $(\mathrm{p}<0.01) . \mathrm{N}_{0}$ : number of individuals sampled in the original populations; $\mathrm{N}_{\mathrm{t}}$ : number of individuals sampled in the synthetics.

$\mathrm{C}_{1}\left(\mathrm{G}_{\mathrm{ST}}=19.5 \%\right)$ revealed an increase of $77.3 \%$. Consequently, the synthetics became more divergent. Such allele losses contributed for this differentiation.

The genetic differentiation $\left(\mathrm{G}_{\mathrm{ST}}\right)$ for the combinations BR-105 vs. IG-3, and BR-106 vs. IG-4 was greater for the first group $(12.4 \%$ vs. $6.8 \%)$ in which the selection intensity was higher $(2.0 \% v s .2 .5 \%)$. These $\mathrm{G}_{\mathrm{ST}}$ values were statistically significant. The $\mathrm{R}_{\mathrm{ST}}$ values were slightly superior to the $\mathrm{G}_{\mathrm{ST}}$ values. As pointed out by Gaiotto et al. (2001) the $\mathrm{R}_{\mathrm{ST}}$ statistic can be used as evidence of genotyp- 
Table 4 - Mean values of gene diversity between the original populations BR-105 and BR-106 and their synthetics IG-3 and IG-4 after one cycle of RRS.

\begin{tabular}{lccccc}
\hline & $\mathrm{H}_{\mathrm{S}}\left(\mathrm{CI}_{95 \%}\right)$ & $\mathrm{H}_{\mathrm{T}}\left(\mathrm{CI}_{95 \%}\right)$ & $\mathrm{D}_{\mathrm{ST}}$ & $\mathrm{G}_{\mathrm{ST}}\left({ }^{*} \mathrm{CI}_{95 \%}\right)$ & $\mathrm{R}_{\mathrm{ST}}\left(* \mathrm{CI}_{95 \%}\right)$ \\
\hline BR-105 vs. BR-106 & 0.57 & 0.64 & 0.07 & $11 \%$ & $11.4 \%$ \\
& $(0.49 ; 0.64)$ & $(0.57 ; 0.71)$ & & $(0.08 ; 0.14)$ & $(0.10 ; 0.13)$ \\
IG-3 vs. IG-4 & 0.46 & 0.58 & 0.11 & $19.5 \%$ & $19 \%$ \\
& $(0.39 ; 0.54)$ & $(0.50 ; 0.65)$ & & $(0.14 ; 0.26)$ & $(0.17 ; 0.22)$ \\
BR-105 vs. IG-3 & 0.50 & 0.57 & 0.07 & $12.4 \%$ & $11 \%$ \\
& $(0.43 ; 0.57)$ & $(0.50 ; 0.64)$ & & $(0.09 ; 0.16)$ & $(0.09 ; 0.13)$ \\
BR-106 vs. IG-4 & 0.53 & 0.57 & 0.04 & $6.8 \%$ & $6.6 \%$ \\
& $(0.46 ; 0.60)$ & $(0.50 ; 0.64)$ & & $(0.05 ; 0.09)$ & $(0.06 ; 0.08)$ \\
\hline
\end{tabular}

*Bootstrap with 10.000 replicates.

ing accuracy due to the fact that its estimation is based on the magnitude of the variances.

\section{Effective population size}

The values for the effective population sizes estimated for the synthetics IG-3 (3.87) and IG-4 (6.62) were similar to those theoretically expected, i.e. for the recombination of 8 and ten $\mathrm{S}_{3}$ lines, respectively (Table 5). In practical terms, it shows that the samples (on average 93 individuals) represent approximately 3.87 and 6.62 plants of an ideal panmitic population and correspond to $4.16 \%$ and $7.11 \%$ of the total sampled individuals from IG-3 and IG-4, respectively. The estimated effective population sizes provided inbreeding coefficients $[(\mathrm{F}=1 / 2 \mathrm{Ne}) .100]$ of $12.91 \%$ for IG-3 and $7.55 \%$ for IG-4, which are similar to the expected values of $10.94 \%$ and $8.75 \%$, respectively.

\section{Discussion}

The differences in the allele frequency distributions between the original populations supported the existence of genetic divergence reported by Naspolini Filho et al. (1981) because of the magnitude of the heterosis manifested in the interpopulation cross.

Changes in allele frequencies between populations and respective synthetics were observed by the lack of overlapping of the confidence intervals in nearly $50 \%$ of the alleles. At most of the loci, the nature of these changes was due to stochastic processes i.e., genetic drift and sampling errors. Nevertheless, changes at some loci were highly significant, and therefore were not due to effects of drift alone.

The apparent lack of neutrality in RRS programs was verified by Labate et al. (1999) at 17\% of the RFLP loci dis-
Table 5 - Effective population size ( $\hat{N} e$ ) for the maize synthetics IG-3 and IG-4. derived from BR-105 and BR-106. respectively.

\begin{tabular}{lcccc}
\hline Synthetics & $\left(\mathrm{N}_{0} ; \mathrm{N}_{1}\right)$ & $\hat{\bar{F}}$ & $\hat{N} e$ & $\mathrm{CI}_{95 \%}$ \\
\hline IG-3 & $(93.50 ; 93.80)$ & 0.14 & 3.87 & $2.90 ; 5.42$ \\
IG-4 & $(93.90 ; 93.50)$ & 0.09 & 6.62 & $5.01 ; 9.14$ \\
\hline
\end{tabular}

$\hat{\bar{F}}$ : mean standardized variance in allele frequency weighted over loci. $\mathrm{N}_{0} ; \mathrm{N}_{1}$ : number of individuals at the two sampling events: populations and synthetics. respectively.

$\mathrm{CI}_{95 \%}$ : $95 \%$ confidence interval.

persed in the maize genome. This result was interpreted as a selection by genetic hitchhiking. As microsatellite loci represent repetitive and non-coding DNA regions, they apparently are not subject to strong selection pressures (Heath et al., 1993). However, they can be linked to selected loci, and therefore subjected to selection by genetic hitchhiking.

The loss of total gene diversity detected at microsatellite loci $(9.4 \%)$ was similar to the decrease of genetic variance $(8.8 \%)$ obtained for yield (Table 6). A similar result was observed in the BSSS and BSCB1 maize populations in which the loss of genetic diversity assessed at RFLP markers was consistent with the decrease in additive and dominant genetic variance in the BSSS population after 12 RRS cycles (Holthaus and Lamkey, 1995; Labate et al., 1999). Both studies confirmed that diversity, or expected heterozygosity, is proportional to genetic variance (Lacy, 1987).

Despite the intensity of the applied selection, our results showed that the total gene diversity loss was not so large after one cycle of RRS. According to Rezende and Souza Jr. (2000), high-intensity RRS selection in BR-105 and BR-106 caused a significant increase in heterosis

Table 6 - Yield and genetic variance $\left(\sigma_{G}^{2}\right)$ for the original (BR-105 and BR-106) and selected (IG-3 and IG-4) maize interpopulations ${ }^{1}$.

\begin{tabular}{lcccccc}
\hline & BR-105 & BR-106 & IG-3 & IG-4 & BR-105 x BR-106 & IG-3 x IG-4 \\
\hline Yield $^{*}\left(\mathrm{Mg} \mathrm{ha}^{-1}\right)$ & 6.52 & 8.00 & 7.48 & 7.19 & 7.90 & 9.22 \\
$\sigma_{G}^{2}\left(\mathrm{~g} \mathrm{p}^{-1}\right)$ & - & - & - & - & 97.47 & 88.86 \\
\hline
\end{tabular}

${ }^{1}$ Data from Rezende and Souza Jr. (2000).

*Yield refers to unhusked ear weight. 
(25.7\% for grain yield) and improved the cross performance between the synthetics IG-3 and IG-4, but the interpopulation genetic variances did not present significant changes (Table 6). The maintenance of genetic variability has been reported in several long and short-term recurrent selection programs (Bernardo, 1996; Guzman and Lamkey, 1999), and by Labate et al. (1999), using RFLP markers. In addition, the use of large effective population sizes has shown no advantage for maintaining genetic variability for short-term recurrent selection (Guzman and Lamkey, 2000). The increase in the genetic differentiation $\left(\mathrm{G}_{\mathrm{ST}}\right)$ after selection $(77.3 \%)$ i.e., in the genetic divergence between the synthetics is predictable in a RRS program. The maintenance of two separate genetic pools allows different alleles to be fixed in each population and guarantees the heterozygous condition for these loci in the interpopulation hybrids. This situation can maximize heterosis whose expression considers the genetic divergence between populations and the dominance effects attributed to the heterozygous loci (Lamkey and Edwards, 1999).

In our results, microsatellites proved to be very promising for monitoring genetic variability and gave support for the use of the improved synthetics in a next cycle of selection. In agreement with previous studies, short-term recurrent selection does not require large effective population sizes, once the genetic variability is maintained at adequate levels after a high-intensity RRS procedure. We can conclude that the modified RRS process, here investigated, would successfully replace the traditional RRS procedure in maize.

\section{Acknowledgments}

This work was partially supported by FAPESP. L.R.P. was supported by fellowship from CNPq.

\section{References}

Bernardo R (1996) Testcross selection prior to further inbreeding in maize: mean performance and realized genetic variance. Crop Science 36:867-871.

Crow JF (1998) 90 years ago: The beginning of hybrid maize. Genetics 148:923-928.

Gaiotto FA, Grattapaglia D and Vencovsky R (2001) Microsatellite markers for heart of palm Euterpe edulis and E. oleracea Mart. (Arecaceae) Molecular Ecology Notes 1:86-88.

Goodman SJ (1997) $\mathrm{R}_{\mathrm{ST}}$ Calc: a collection of computer programs for calculating estimates of genetic differentiation from microsatellite data and determining their significance. Molecular Ecology 6:881-885.

Goudet J (1995) FSTAT (v. 12): a computer program to calculate F-statistics. Journal of Heredity 86:485-486.

Guzman PS and Lamkey KR (1999) Predicted gains from recurrent selection in the BS11 maize population. Maydica 44:93-99.
Guzman PS and Lamkey KR (2000) Effective population size and genetic variability in the BS11 Maize population. Crop Science 40:338-346.

Hallauer AR and Miranda JB (1988) Quantitative Genetics in Maize Breeding. 2nd ed. Iowa State University Press, Ames, $340 \mathrm{pp}$.

Heath DD, Iwama GK and Devlin RH (1993) PCR primed with VNTR core sequences yields species specific patterns and hypervariable probes. Nucleic Acids Research 21:57825785.

Holthaus JF and Lamkey KR (1995) Population means and genetic variances in selected and unselected Iowa Stiff Stalk synthetic maize populations. Crop Science 35:1581-1589.

Hoisington D, Khairallah M and González-De-León D (1994) Laboratory Protocols: CIMMYT Applied Molecular Genetics Laboratory. CYMMYT, Mexico, p 51.

Jarne P and Lagoda PJL (1996) Microsatellites, from molecules to population and back. Tree 11:424-429.

Koeyer DL, Phillips RL and Stuthman DD (2001) Allelic shifts and quantitative trait loci in a recurrent selection population of oat. Crop Science 41:1228-1234.

Labate JA, Lamkey KR, Lee M and Woodman WL (1997) Molecular genetic diversity after reciprocal recurrent selection in BSSS and BSCB1 maize populations. Crop Science 37:416-423.

Labate JA, Lamkey KR, Lee M and Woodman WL (1999) Temporal changes in allele frequencies in two reciprocally selected maize populations. Theoretical and Applied Genetics 99:1166-1178.

Lacy RC (1987) Loss of genetic diversity from managed populations: Interacting effects of drift, mutation, immigration, selection, and population subdivision. Conservation Biology 1:143-158.

Lamkey KR and Edwards JW (1999) The quantitative genetics of heterosis. In: Coors JG, Pandey S, Ginkel MV, Hallauer AR, Hess DC, Lamkey KR, Melchinger AE, and Stuber CW (eds) Proceedings of the International Symposium on the Genetics and Exploitation of Heterosis in Crops. CIMMYT, Mexico City, Mexico, 1997, pp 31-48.

Miller MP (1997) Tools for population genetic analyses (TFPGA) 1.3: a windows program for the analysis of allozyme and molecular population genetic data. http://www.public. asu.edu.

Naspolini Filho V, Gama EEG, Vianna RT and Moro JR (1981) General and combining ability for yield in a diallel cross among 18 maize populations (Zea mays L.). Revista Brasileira de Genética 4:571-577.

Nei M (1973) Analysis of gene diversity in subdivided populations. Proceedings of the National Academy of Sciences of the USA 70:3321-3323.

Nei M and Tajima F (1981) Genetic drift and estimation of effective population size. Genetics 98:625-640.

Ogliari JB, Boscariol RL and Camargo LEA (2000) Optimization of PCR amplification of maize microsatellite loci. Genetics and Molecular Biology 23:395-398.

Rezende GDSP and Souza Jr. CL (2000) A reciprocal recurrent selection procedure outlined to integrate hybrid breeding programs in maize. Journal of Genetics and Breeding 54:57-66.

Slatkin M (1995) A measure of population subdivision based on microsatellite allele frequencies. Genetics 139:457-462. 
Souza Jr. CL (1998) Seleção recorrente e desenvolvimento de híbridos. Reunión Latino Americana de Investigadores en Maiz. Santa Fé de Bogotá, Colombia, 1998, pp 18-19.

Swoffford DL and Selander, RB (1991) Biosys-1: a FORTRAN program for the comprehensive analysis for electrophoretic data in population genetics and systematics. Journal of Heredity $38: 1358-1370$.
Waples RS (1989a) Temporal variation in allele frequencies: testing the right hypothesis. Evolution 43:1236-1251.

Waples RS (1989b) A generalized approach for estimating effective population size from temporal changes in allele frequency. Genetics 121:379-391.

Wright S (1951) The genetical structure of populations. Annals of Eugenetics 15:313-354.

Editor: Marcio de Castro Silva-Filho 